\title{
DUINO-BASED LEARNING (DBL): UN PROYECTO PARA FACILITAR EL USO DE ARDUINO Y MATLAB
}

\author{
Eneko Lerma, Robert Griñó, Ramon Costa-Castelló \\ Departamento de Ingeniería de Sistemas, Automática e Informática Industrial (ESAII) \\ Universitat Politècnica de Catalunya (UPC) \\ e-mail: eneko.lerma@estudiant.upc.edu, roberto.grino@upc.edu, ramon.costa@upc.edu \\ Carlos Sanchis \\ Mathworks, e-mail: Carlos.Sanchis@mathworks.es
}

\section{Resumen}

En este trabajo se presenta un conjunto de puntos de partida para llevar a cabo un aprendizaje basado en proyectos, ya que, normalmente, esta metodología suele ser difícil de implementarla desde cero. Duino-Based Learning (DBL) es un proyecto que ofrece material distribuido libremente para llevar a cabo proyectos de laboratorio mediante MATLAB, Simulink, y Arduino. En el trabajo se muestra como estos materiales han sido aplicados al desarrollo de las prácticas de control digital utilizando la planta didáctica módulo LJ Technical Systems.

Palabras clave: Arduino, Simulink, MATLAB, acondicionamiento de señales, Aprendizaje basado en proyecto.

\section{INTRODUCCIÓN}

Este proyecto nace de la necesidad de actualizar el laboratorio de control automático de la Escola Tècnica Superior d'Enginyeria Industrial de Barcelona (ETSEIB) de la Universitat Politècnica de Catalunya (UPC). En este laboratorio los alumnos aprenden control en tiempo continuo y tiempo discreto. Durante las sesiones, los estudiantes implementan lo que han aprendido en las clases teóricas y se utiliza el módulo LJ Technical Systems (ver Figura 1) como planta de trabajo. Esta planta corresponde a un motor de corriente continua junto con sus etapas de acondicionamiento de señales y potencia. El módulo contiene los elementos de medida necesarios para cerrar los lazos de control de velocidad, gracias al dinamo taquimétrica, y de posición, gracias al potenciómetro. Además de esto, este motor está equipado con un freno magnético, el cual se usa como perturbación.

Antes de comenzar este proyecto, los estudiantes adquirían los datos desde las tarjetas $\mathrm{AD} / \mathrm{DA}$ integradas en los ordenadores del laboratorio. Estas tarjetas son caras y utilizan el bus PCI, que cada vez es menos común, por lo que complica las actualizaciones de los equipos del laboratorio.

En los últimos tiempos han aparecido diferentes equipos que pueden ser fácilmente utilizables en

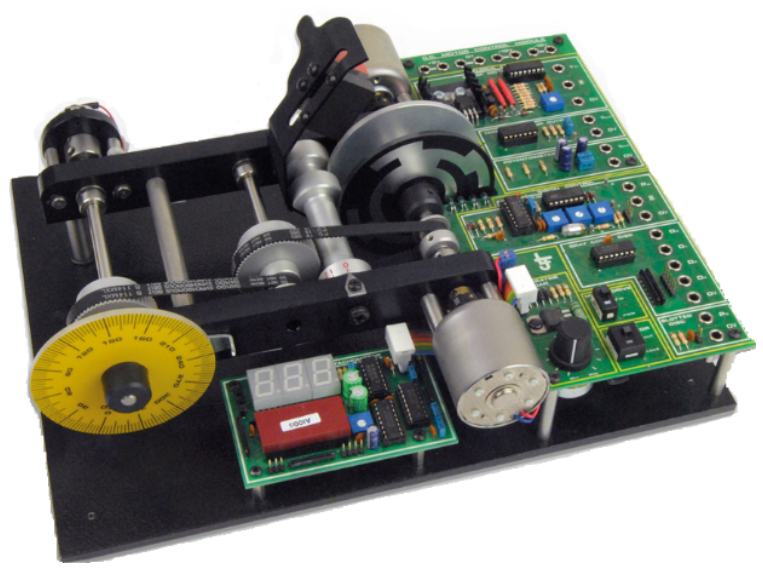

Figura 1: Módulo LJ TechnicalSystems

la realización de prácticas de control automático [2, 3, 10]. Ello ha hecho reconsiderar los equipos que se van a utilizar en la definición de los nuevos puestos de trabajo. Por ello se tomó la decisión de cambiar los puestos de trabajo actuales, por dispositivos basados en Arduino [9]. De entre toda a gama de modelos Arduino existentes se eligió el Arduino Due, debido a tres razones principales:

- Es uno de los pocos modelos equipados con elementos de conversión digital-analógico (DAC) reales

- Presenta hasta 12 canales de entrada analógica con una resolución de 12 bits, mientras que otros modelos más conocidos, como el Arduino Uno o Arduino Mega, solo tienen 10 bits de resolución.

- El Arduino Due tiene un alto rendimiento el cual permite una mayor velocidad de cómputo, lo que mejora la aplicación de un sistema de control digital.

Aunque las placas basadas en Arduino presentan su propio entorno de programación, en la actualidad es posible programar dichas placas mediante generación automática de código desde MATLAB/Simulink 8. Ello es de gran interés para la realizar prácticas de control automático, pues permite a los estudiantes centrarse en los concep- 


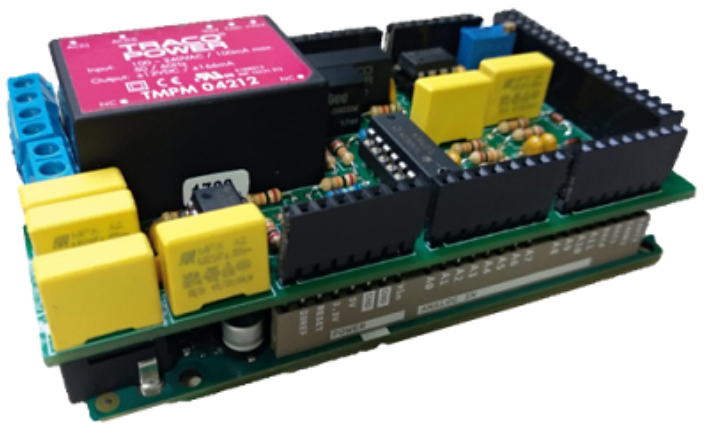

Figura 2: Arduino Due junto con la placa de acondicionamiento de señal.

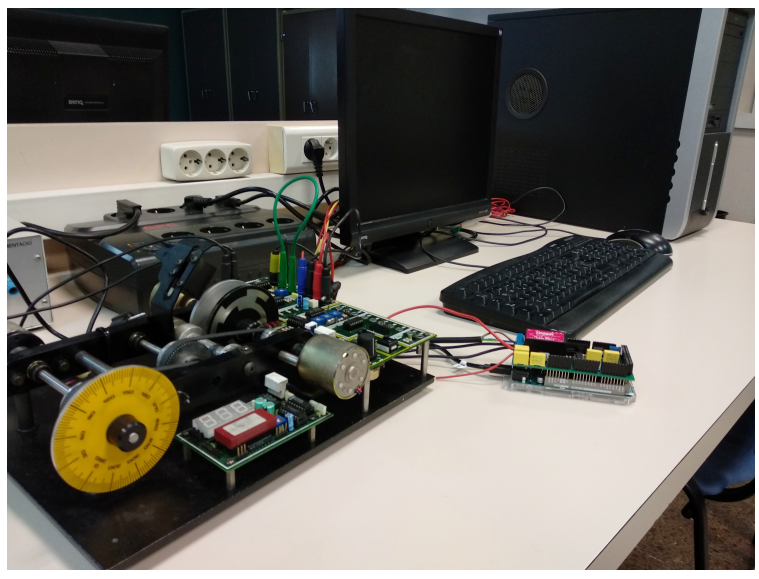

Figura 3: Una de las estaciones de trabajo del laboratorio de control automático.

tos de control y no en los vinculados a la codificación de los controladores.

El trabajo se organiza de la siguiente manera, la sección 2 contiene una descripción del proyecto desarrollado, y finalmente la sección 3 presenta la conclusiones del trabajo.

\section{DUINO-BASED LEARNING (DBL)}

Después de haber remodelado el hardware y el software del laboratorio satisfactoriamente [8], se decidió crear una página web, donde se describen los pasos seguidos durante el proyecto, junto a diferentes vídeo tutoriales y ejercicios propuestos, para que cualquiera que quiera hacer un proyecto tenga un punto de partida y una base donde poder amarrarse.

Con el objetivo de hacer compatibles los rangos de tensión de las E/S del Arduino Due y los del módulo LJ Technical Systems, se creó un shield (ver Figure 2) encargado de acondicionar dichas tensiones. Este circuito, puede fácilmente adaptarse a otros niveles de tensión utilizando los elementos incorporados en él (resistencias y condensado-

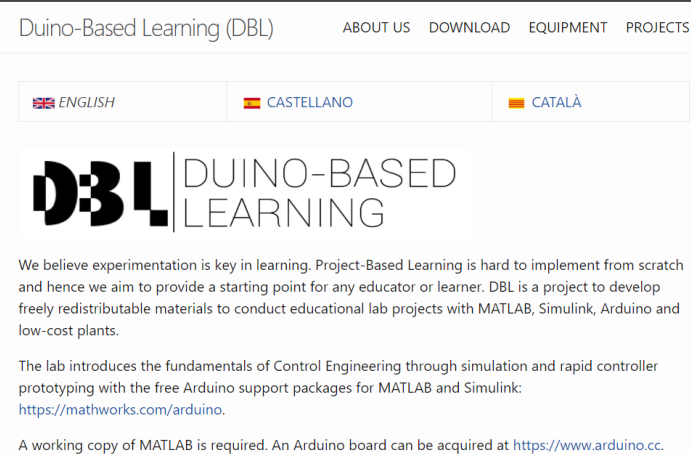

Projects

The practices of the subject Computer Control consist in the study of a servomechanism of angular positioning (LU Technical Systems) controlled by a PC. The laboratory sessions P1 and P2 focus on the experimental analysis of the temporal and frequency responses of the system respectively. Sessions $\mathrm{P}$ and P4 are dedicated to the design of PID controllers. Finally, in practice P5 controllers will be designed

Figura 4: Página de inicio de Duino-Based Learning.

res). Se podría decir, que se ha creado un shield universal especialmente para el modelo Arduino Due, para adaptar rangos entre la misma placa y una planta a controlar (los esquemáticos pueden ser descargados directamente de la página web del proyecto).

Después de un proceso de diseño y validación mediante simulación y prototipos preliminares. Actualmente, se dispone del equipo en formato final (Figura 2). La Figura 3, muestra el estación de trabajo completa en el formato actual. Toda la información sobre los diferentes pasos seguidos y los componentes está disponible en una página web en tres idiomas (inglés, español y catalán). Dicha página contiene además un repositorio de diferentes ejemplos y códigos que pueden ser utilizados para montar nuevas prácticas y desarrollar proyecto. A esta web (Figura 4) le hemos dado el nombre de Duino-Based Learning: https://github.com/DuinoBasedLearning/Lab

\subsection{El proyecto}

La puesta en marcha de asignaturas que utilizan el aprendizaje basado en proyectos suele requerir un esfuerzo muy elevado. Pues suele es complicado empezar los proyectos desde cero. Pese a ello es una excelente manera de que los estudiantes pongan en práctica todas las tecnologías aprendidas em el aula y puedan ser conscientes de las diferencias entre la teoría y la realidad. Disponer de herramientas base que faciliten la puesta en marcha es una manera de reducir dichos esfuerzos.

Con esta idea en mente, se puso en marcha el proyecto Duino-Based Learning. Este proporciona un conjunto de puntos de partida para proyectos que puede ser útil para cualquier educador o estudiante. En él puede encontrar instrucciones 
de compilación para todas las configuraciones, secuencias de comandos en vivo de MATLAB con ejercicios (MATLAB live scripts), modelos de Simulink y vídeo tutoriales. Todo este material está disponible en tres idiomas, inglés, español y catalán. La página web se puede encontrar en https://duinobasedlearning.github.io/.

La Figura 4 muestra el diseño de la pàgina web que contiene toda la información. Como puede verse, la página web está dividida en 5 secciones. La primera es la página de inicio, donde se incluye toda la información principal, la segunda pestaña es la sección donde se presentan a los autores del proyecto. La siguiente, download, contiene la descripción de como descargar y ejecutar los archivos disponibles en el repositorio. En la sección equipment se explica toda la instrumentación utilizada para llevar a cabo los proyectos y cómo, los diferentes elementos, están conectados entre sí. Finalmente, en la última pestaña, puede encontrar cinco proyectos basados en BDL desarrollados para las prácticas de la asignatura Control Automático de la ETSEIB, con instrucciones para construir plantas, ejercicios y vídeos de bajo costo, consulte la Figura 5. Esta es una asignatura donde los estudiantes son introducidos en el mundo del control digital [5] [4] 7].

Para cada unas se la prácticas, se ofrecen los scripts de MATLAB junto con datos, gráficos y explicaciones, mientras que el segundo corresponde a los vídeos explicativos, donde se muestran unas breves píldoras conceptuales, el desarrollo de la práctica y el comportamiento del motor durante el desarrollo de los experimentos controladores desarrollados. En esta última pestaña se ha añadido un video adicional, donde se explica los controles adaptativos indirectos implementados para el caso de control de velocidad y para el caso de control de posición. Ello ha permitido implementar un regulador autoajustable [1, donde primeramente se estiman los parámetros del proceso mediante el algoritmo de identificación por mínimos cuadrados recursivos (RLS) y a partir de esta estimación, se actualizan las ganancias del controlador.

\subsection{Ejemplos de uso}

Como se ha mencionado anteriormente, a lo largo de toda la página web hay cinco prácticas diferentes, las cuales se llevan a cabo en el laboratorio que el departamento de ingeniería de sistemas (ESAII) de la UPC tiene en la ETSEIB. La descripción de estas experiencias se complementa con algunos ejercicios propuestos y vídeo tutoriales. Desde la introducción muy básica a la programación de Arduino a través de MATLAB/Simulink, hasta el
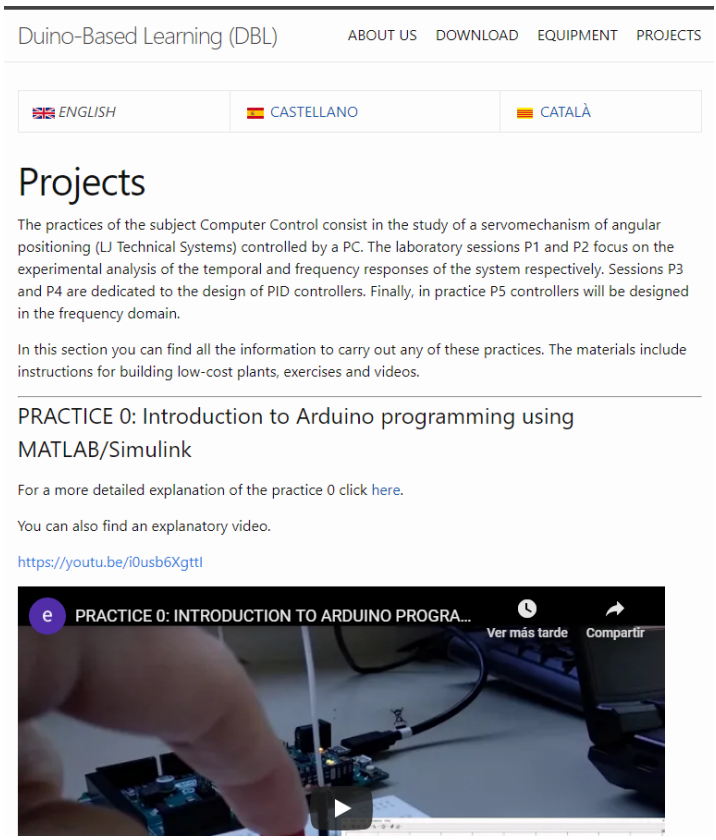

Figura 5: Sección de proyectos de Duino-Based Learning.

diseño de controladores en el dominio de la frecuencia. Además de esto se ha incluido un vídeo tutorial explicando el control adaptativo indirecto implementado como estudio adicional. La Figura 5 muestra el diceño de la pestaña dedicada a los proyectos.

\subsubsection{Introducción a la programación en Arduino mediante MATLAB/Simulink}

El objetivo principal de esta primera práctica es aprender cómo configurar Simulink para permitir la conexión y la transferencia de datos entre la placa Arduino y Simulink. Junto con esto, se explica el uso de los bloques de entrada y salida analógicos y digitales específicos de Arduino. La Figura 6 muestra el gráfico obtenido al conectar un botón al modelo de Digital Input de Simulink.

La documentación y en vídeo incluye los siguientes elementos : Digital output, Digital input, Analog output y Analog input, que posteriormente son necesarios para la realización del resto de prácticas.

\subsubsection{Análisis de la Respuesta Temporal de un Sistema de Control Digital}

En este segundo paquete de trabajo, vamos a analizar la respuesta temporal del servo sistema de LJ Technical Systems. En primer lugar, obtendremos un modelo de comportamiento basado en el estudio de la respuesta temporal de lazo abierto. Luego cerraremos un lazo de control proporcional en salida velocidad y analizaremos el efecto de la ganancia proporcional en la precisión y estabili- 


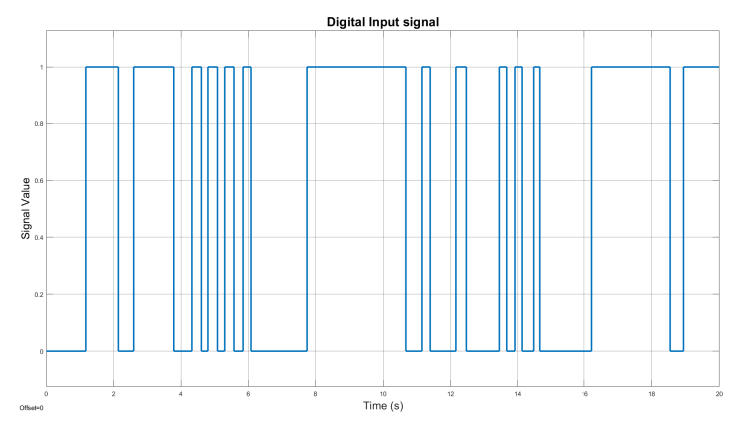

Figura 6: Señal obtenida en uno de los experimentos de la práctica 0 .

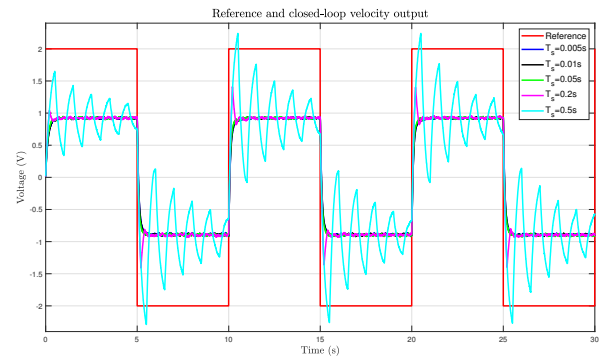

Figura 7: Efecto del período de muestreo para el caso de velocidad.

dad del sistema. También analizaremos el efecto del tiempo de muestreo en la estabilidad del sistema de lazo cerrado, muestreando con diferentes periodos de muestreo. Se seguirán los mismo pasos para el control de posición. Los pasos seguidos son los siguientes:

1. Control de velocidad

- Respuesta de lazo abierto

- Respuesta de lazo cerrado

- Evaluación experimental de las prestaciones del sistema en lazo cerrado

2. Control de posición

- Respuesta de lazo abierto

- Respuesta de lazo cerrado

- Evaluación experimental de las prestaciones del sistema en lazo cerrado

La Figura 7 muestra la evaluación experimental de la velocidad del sistema en lazo cerrado de velocidad para diferentes valores del período de muestreo y un mismo valor de la ganancia proporcional.

\subsubsection{Análisis de la respuesta frecuencial de un sistema de control digital}

En esta práctica se analiza la respuesta en frecuencia del sistema LJ Technical Systems. Aquí se obtenedrá el diagrama de Nyquist experimental y se comparara con la teórica (obtenida a partir de

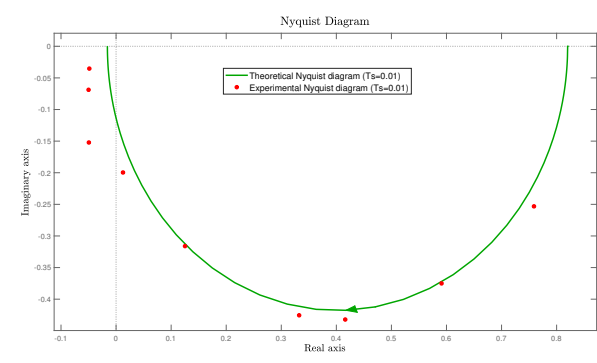

Figura 8: Comparación entre el diagrama de $\mathrm{Ny}$ quist ideal y el experimental.

la función de transferencia ajustada en la práctica anterior). Junto con esto, aplicaremos el criterio de Nyquist para determinar el rango de valores de un controlador proporcional para que el sistema sea estable y se verificará experimentalmente.

1. Obtención del diagrama de Nyquist

2. Obtención experimental del diagrama de Nyquist del sistema discreto

3. Aplicación del criterio de Nyquist

4. Obtención del diagrama teórico de Bode

La Figura 8 muestra la comparación entre el diagrama de Nyquist experimental y el teórico.

\subsubsection{Diseño e implementación de controladores PID}

La tercera práctica trata de diseñar controladores PID. En primer lugar, se diseñará un controlador PI digital para velocidad y, posteriormente, se diseñará un PID digital para la posición.

1. Diseño e implementación de un PI por asignación de polos para salida velocidad

2. Diseño e implementación de un PID por asignación de polos para salida posición

\subsubsection{Mejora de los controladores PID}

Como en la práctica anterior no se tienen en cuenta posibles cancelaciones de ceros, o posibles mejoras en la implementación, en esta práctica, se presenta un posible cambio para la mejora de la dinámica del sistema en lazo cerrado, modificando para ello la arquitectura actual de los controladores y diseñando así, un controlador I-P para velocidad y un controlador I-PD para posición.

1. Diseño e implementación de un I-P por asignación de polos para salida velocidad

2. Diseño e implementación de un I-PD por asignación de polos para salida posición 


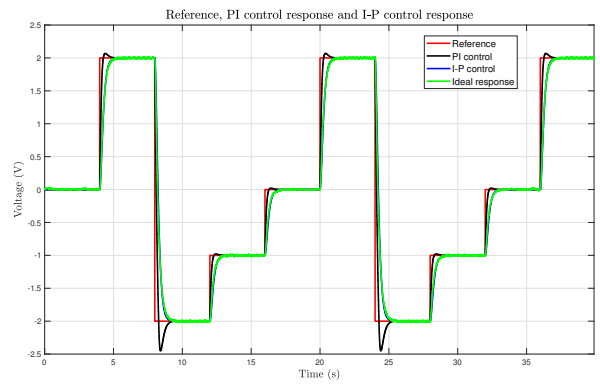

Figura 9: Comparación experimental de los sistemas de control PI e I-PD.

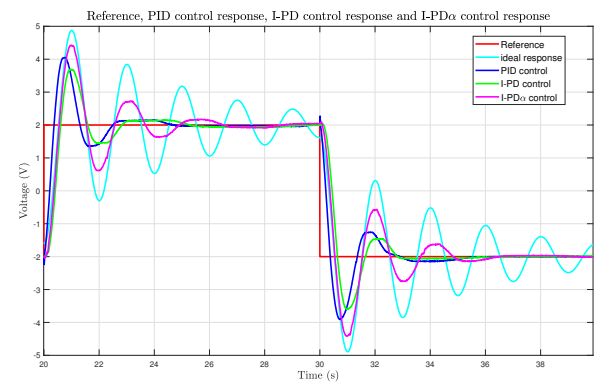

Figura 10: Comparación experimental de los sistemas de control PID, I-PD e I-PD $\alpha$.

Las Figuras 9 y 10 muestran los controladores implementados en la práctica actual junto a la comparación con los resultados de la práctica 3 .

\subsubsection{Diseño de controladores en campo frecuencial}

En este quinto trabajo, veremos cómo diseñar un controlador en el campo frecuencial, de modo que podamos modificar el comportamiento del sistema en lazo cerrado. Junto a esto, se diseñará un controlador por avance de fase para que la respuesta del motor en lazo cerrado, para el control de posición, presente un error estacionario del $10 \%$ frente a una entrada tipo rampa como se puede analizar en la Figura 11 .

1. Determinación de los márgenes de ganancia y fase del sistema

2. Diseño de un controlador de avance de fase.

3. Análisis de simulación

4. Implementación del controlador de avance de fase.

\subsubsection{Control Adaptativo Indirecto}

Como se ha comentado anteriormente, en esta última práctica se estudian e implementan dos controladores autoajustables, uno para el control de velocidad y otro para el control de posición. Los parámetros de la planta se estiman mediante el

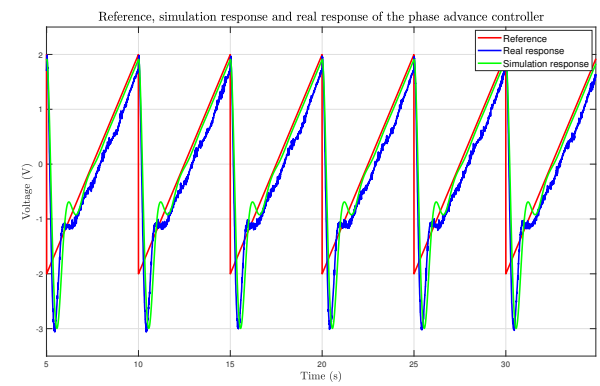

Figura 11: Respuesta del sistema a una entrada de tipo rampa con controlador de avance de fase.

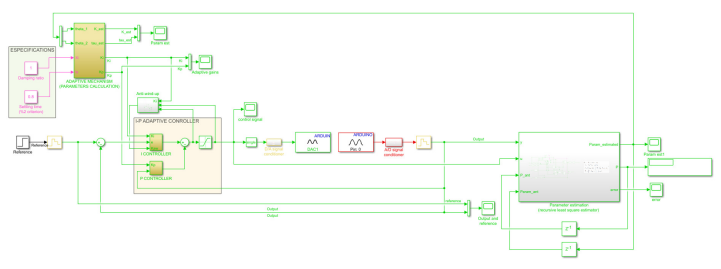

Figura 12: Modelo de Simulink implementado para el controlador I-P adaptativo indirecto.

algoritmo de mínimos cuadrados recursivos [6] los cuales hacen que se actualicen las ganancias del controlador I-P o I-PD, dependiendo del ejercicio. La Figura 12 muestra el diagrama de bloques implementado en Simulink para el caso de velocidad

1. Diseño e implementación de un PI adaptativo inirecto por asignación de polos para salida velocidad

2. Diseño e implementación de un PID adaptativo inirecto por asignación de polos para salida posición

\subsection{REPOSITORIO}

Todos los modelos implementados en la página web están disponibles gratuitamente en GitHub junto a los archivos Gerber utilizados para la implementación de la placa, un pdf con la explicación de los pasos que se han seguido para su creación y los Live Scripts de MATLAB en los tres idiomas mencionados. El objetivo de este proyecto, junto con ayudar a los alumnos a comprender los conceptos básicos del control digital o ayudar a los educadores a la hora de idear algunas lecciones prácticas, es animar a las personas a contribuir con nuevas ideas o mejores implementaciones y así obtener una actualización continua de la página web. El repositorio se puede encontrar en https://github.com/DuinoBasedLearning/Lab. 


\section{CONCLUSIONES}

En este trabajo se presentan los contenidos y el material didáctico utilizado en un curso de control de tiempo discreto. Todo el material está disponible y puede ser libremente utilizado. Todo el trabajo se basa en el uso de herramientas de diseño de control interactivo, como MATLAB, para ayudar a los alumnos a comprender qué es un sistema de datos muestreado y cómo manejar su control. Se proponen experimentos basados en hardware de bajo costo como Arduino que puede alentar a las personas a ingresar al mundo del control digital.

Este documento puede ser un ejemplo de cómo se podría enseñar o aprender este tema sin estar demasiado concentrado en los cálculos.

\section{Agradecimientos}

This work has been partially funded by the Spanish national projects MICAPEM (ref. DPI2015-69286-C3-2-R, MINECO/FEDER), DPI2017-85404-P and the donation of Mathworks UPC-I-01523.

\section{English summary}

\section{DUINO-BASED LEARNING (DBL): A PROJECT TO SIM- PLIFY THE USE OF ARDUINO AND MATLAB}

\begin{abstract}
This paper presents a set of starting points to carry out a project-based learning, since this methodology is usually difficult to implement from scratch. Duino-Based Learning $(D B L)$ is a website where you can find freely redistributable materials to carry out laboratory projects using MATLAB, Simulink, Arduino and low cost plants, such as the LJ Technical Systems module, which has been used in this work.
\end{abstract}

Keywords: Arduino, Simulink, MATLAB, signal conditioning, DC motor control, Project-based learning.

\section{Referencias}

[1] Karl Johan Astrom and Bjorn Wittenmark. Adaptive Control. Addison-Wesley Longman Publishing Co., Inc., Boston, MA, USA, 2nd edition, 1994.

[2] R. Barber, M. Horra, and J. Crespo. Practices using simulink with arduino as low cost hardware. IFAC Proceedings Volumes, 46(17):250 - 255, 2013.

[3] F.A. Candelas, G.J. García, S. Puente, J. Pomares, C.A. Jara, J. Pérez, D. Mira, and F. Torres. Experiences on using arduino for laboratory experiments of automatic control and robotics. IFAC-PapersOnLine, 48(29):105 - 110, 2015.

[4] Oriol Causí Casamor, Miquel Angel Mañanas Villanueva, Ramon Costa Castelló, and Luis Basañez Villaluenga. Control amb Computador. Simulación en entorno MATLAB. CPDA, Barcelona, Octubre 1999. ISBN 84-95355-04-3.

[5] Ramon Costa Castelló and Enric Fossas. Sistemes de Control en Temps Discret. Edicions UPC, 2014. ISBN: 978-84-9880-492-8.

[6] José de Jesús Rubio, Enrique García, Gustavo Aquino, Carlos Aguilar-Ibáñez, Jaime Pacheco, and Jesús Alberto Meda-Campaña. Mínimos cuadrados recursivos para un manipulador que aprende por demostración. Revista Iberoamericana de Automática e Informática industrial, 16(2):147-158, 2019.

[7] Profesores de la asignatura. Control por computador: Pràcticas. Technical report, Escuela Técnica Superior de Ingeniería Industrial de Barcelona (ESTEIB), Barcelona, 1998.

[8] Eneko Lerma, Robert Griñó, Ramon CostaCastelló, and Carlos Sanchis. Implementación de controladores en arduino mediante simulink. In UNIVERSIDAD DE EXTREMADURA, editor, Actas de las XXXVIII Jornadas de Automática, pages 158-164, Badajoz,, 5-7 de Septiembre de 2018 2018. CEA.

[9] Alejandro Pérez Ruiz, Mario Aldea Rivas, and Michael González Harbour. Aplicaciones ada en android con requisitos de tiempo real. Revista Iberoamericana de Automática e Informática industrial, 16(3):264-272, 2019.

[10] Jaroslav Sobota, Roman PiSl, Pavel Balda, and MiloS Schlegel. Raspberry pi and arduino boards in control education. IFAC Proceedings Volumes, 46(17):7 - 12, 2013.

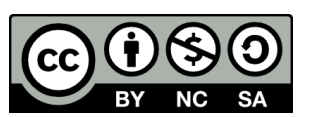

(C) 2019 by the authors. Submitted for possible open access publication under the terms and conditions of the Creative Commons Attribution CC BY-NC-SA 4.0 license (https://creativecommons.org/licenses/by-ncsa/4.0/deed.es). 